\title{
Contrast CT Examination for Assessment of Intestinal Bleeding in a Polytraumatized Cat - Case Report
}

\author{
Radu LĂCĂTUŞ ${ }^{1}$, Robert Cristian PURDOIU ${ }^{*}$, Alin ORĂȘAN ${ }^{2}$, Filip ARDELEAN ${ }^{3}$, Ileana MATEI ${ }^{3}$, Ionel \\ PAPUC $^{1}$ \\ ${ }^{1}$ University of Agricultural Sciences and Veterinary Medicine, Faculty of Veterinary Medicine Department \\ of Semiology, Radiology and Medical Imaging, Mănăștur, no. 3-5, Cluj Napoca, Romania; \\ ${ }^{2}$ Emergency Service Cluj Napoca. University of Medicine and Pharmacy "Iuliu Hațieganu" Cluj Napoca \\ ${ }^{3}$ Department of Reconstructive Surgery. Recovery Clinical Hospital Cluj-Napoca. \\ *Corresponding author: robert.purdoiu@usamvcluj.ro
}

Bulletin UASVM Veterinary Medicine 73(1) / 2016,

Print ISSN 1843-5270; Electronic ISSN 1843-5378

DOI:10.15835/buasvmcn-vm: 11964

\begin{abstract}
Accident could occur often and cats could be subjected to them. Examining a polytraumtized cat could present a challenge for the vet, being very easy to miss a small active bleeding, especially when that hemorrhage is located in the intestinal lumen. The aim of this case study was to highlight a possible hemorrhagia in the gastrointestinal tract, in the arterial time of contrast CT in a polytraumatized cat.

A 2 year, mixed breed cat was brought for radiography examination after it was hit by a car. Radiographic exposures were done for the spine and the abdomen and ultrasound examination was used to check for internal bleeding. Contrast agent Visipaque (iodixanol, $320 \mathrm{mgl} / \mathrm{ml}$, producer Nycomed Amersham) was administered using automated injection after the patient was general anesthetized, the dose being adjusted by the device.

Clinical examination showed paleness of the mucosa and paralysis of the hind legs. Classic radiographic examination showed a luxation of the spine in the thoracic-lumbar area, and a normal aspect of the abdominal organs. The ultrasound do not reveal liquid in the abdominal cavity. Contrast CT revealed an accumulation of the contrast agent in the intestinal lumen in the arterial time of the procedure.

Contrast CT examination proved to be extremely useful and revealed an accumulation of the contrast agent in the intestinal lumen due to intestinal bleeding which explain the paleness of the mucosa in our patient. Radiography and ultrasonography have not proven reliable in this case for a diagnostic of internal bleeding.
\end{abstract}

Keywords: Cat, Contrast CT, bleeding, intestinal haemorrhage, polytrauma

\section{INTRODUCTION}

Accident could occur often and cats could be subjected to them. Examining a polytraumatized cat could present a challenge for the vet, being very easy to miss a small active bleeding, especially when that hemorrhage is located in the intestinal lumen. Gastro-intestinal tract hemorrhagia and traumatism could represent causes for sepsis (Grassi et al., 1998). Because of the fluid content in the gastrointestinal tract, small quantity of blood or an active small hemorrhagia could easily be missed
(Boysen et al., 2003). Despite that Computed Tomography examination is not available in every clinic, being also an expensive tool, compared with ultrasonography and radiography, it can offer additional information in case of abdominal trauma (Kleine and Lamb, 1989).

Different studies have described the utilization of CT as a Para clinical survey method in veterinary medicine (Moore et al., 1991; Burk, 1992; Plummer et al., 1992; Patsikas et al., 2001; Sojaei et al., 2006). While radiographic examination 
is subjected to artefact because the abdominal fat tissue in the cat abdomen (Heng et al, 2005), contrast CT could highlight the organs and possible hemorrhagia (Schwarz et al., 2011). Accuracy of helical multi-slice CT studies relay upon image acquisition during different enhancement phases after contrast agent's injection (Bae, 2010; Makara et al., 2013).

The aim of this case study was to highlight a possible hemorrhagia in the gastrointestinal tract, in the arterial time of contrast CT in a polytraumatized cat.

\section{MATERIALS AND METHODS}

A 2 year, mixed breed cat was brought for radiography examination after it was hit by a car. The clinical examination shows paresis of the back legs, multiple contusions in the cervical area, mild rectal hemorrhagia. The cat was conscious during clinical evaluation.

Radiographic exposures were done in order to examine the spine and the pelvic area, and abdominal ultrasound examination, using a linear probe of 7.5-10 Mhz, was performed to check for internal bleeding.

Computed Tomography was performed with the cat undergoing sedation using Diazepam (Barr) $0.5 \mathrm{mg} / \mathrm{kg}$ i.m., Butorfanol (Bristol-Myers) i.v. $0.2 \mathrm{mg} / \mathrm{kg}$, Propofol (Pfizer) $5 \mathrm{mg} /$ animal by intravenous injection.

A native CT using a TAP (Thorax-AbdomenPelvis) window was performed, after which contrast agent was administered i.v. Contrast agent Visipaque (iodixanol, $320 \mathrm{mgl} / \mathrm{ml}$, producer Nycomed Amersham) was administered with the help of automatic injector with a flow of $2.0 \mathrm{ml} /$ sec for a period of 17 seconds at a pressure of 120 psi.

\section{RESULTS AND DISCUSION}

Clinical examination showed pallor of the mucosa and paralysis of the hind legs. Classic radiographic examination shows a luxation of the spine in the thoracic-lumbar area, and a normal aspect of the abdominal organs. The ultrasound do not reveal liquid in the abdominal cavity.

The luxation of the spine was highlighted better on the native CT examination, the vertebrae not only being luxated but also twisted on the long axis of the spine and presented a fracture of the dorsal processes (fig. 1).

After contrast agent administration, the exposure was performed at $5 \mathrm{~s}$, to highlight the arterial time. Contrast CT revealed an accumulation of the contrast agent in the gastrointestinal wall

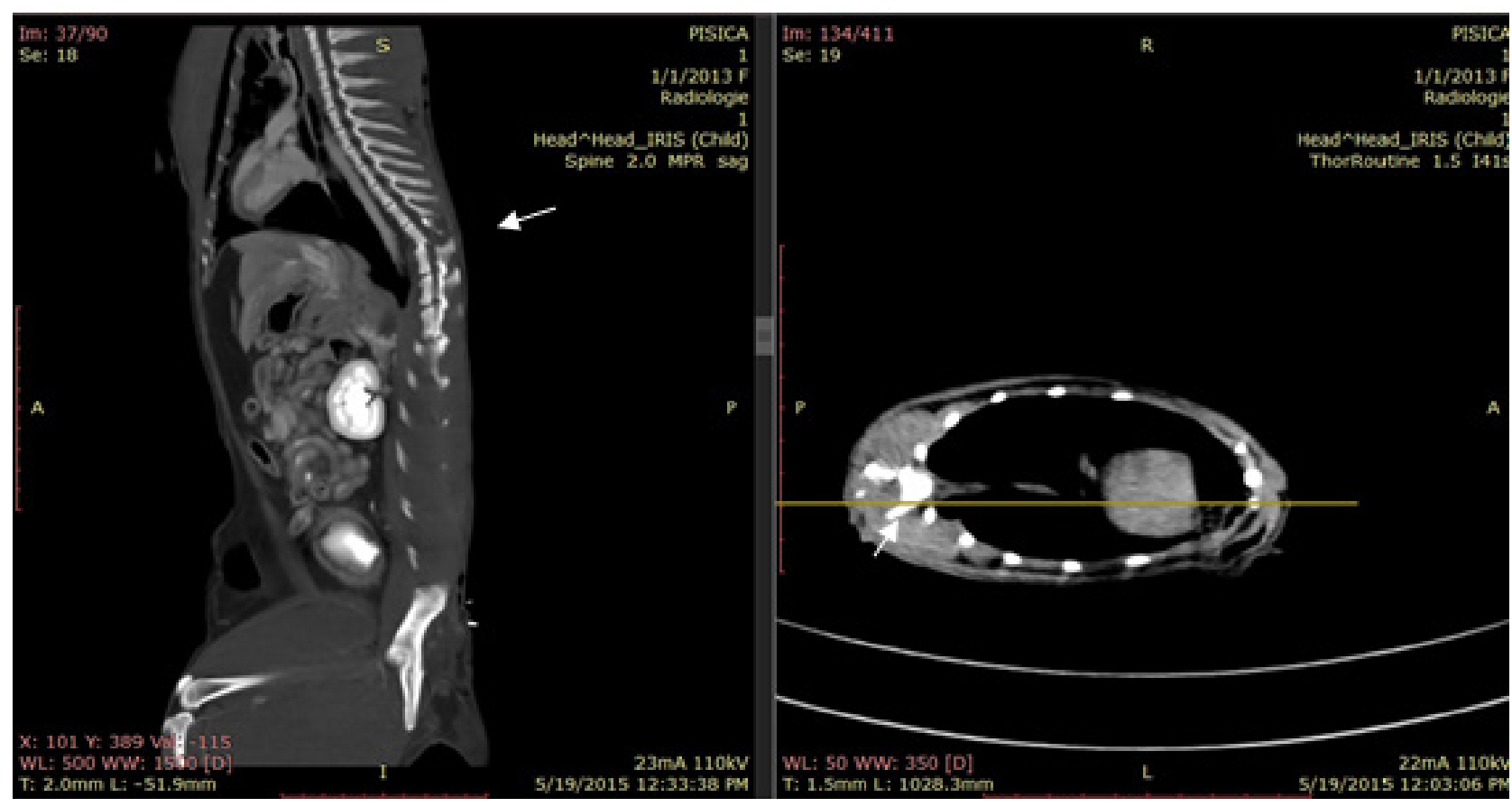

Fig. 1. Sagittal and coronal rendering of the spine, luxation and fracture (white arrow), 110kV, $22 \mathrm{~mA}$ 


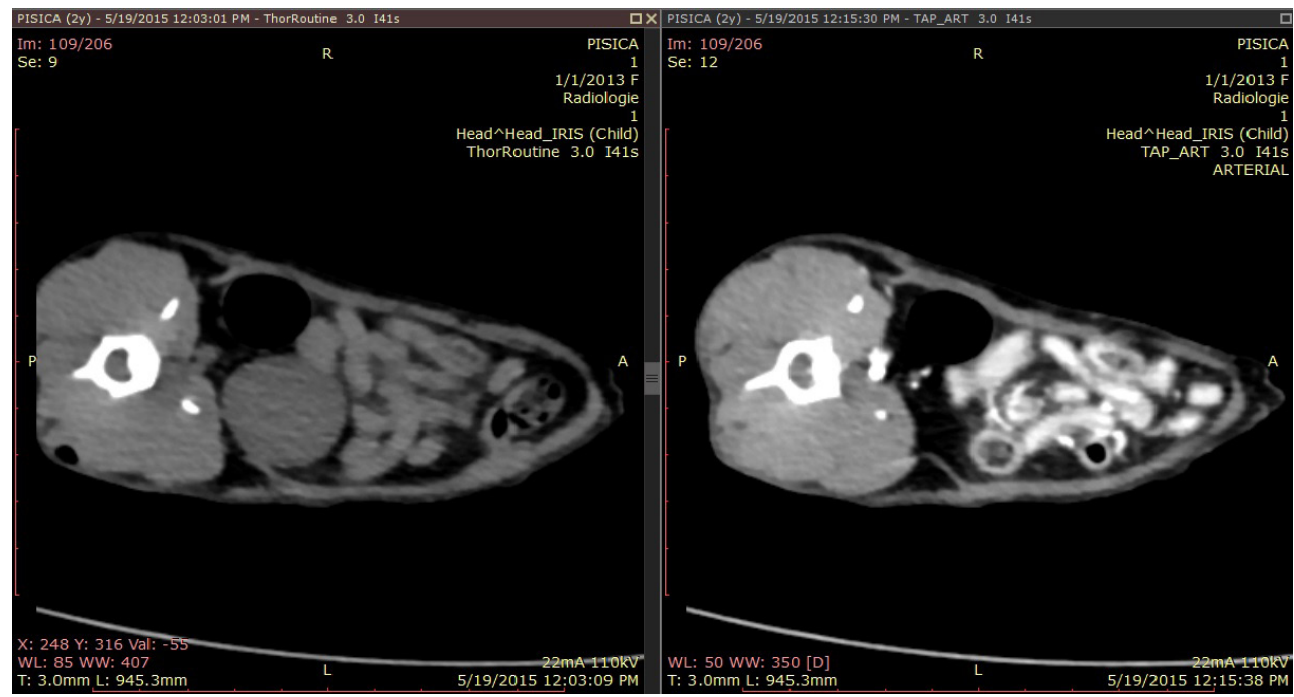

Fig. 2. Pre contrast and post contrast coronal view of the small intestine, $110 \mathrm{kV}, 22 \mathrm{~mA}$

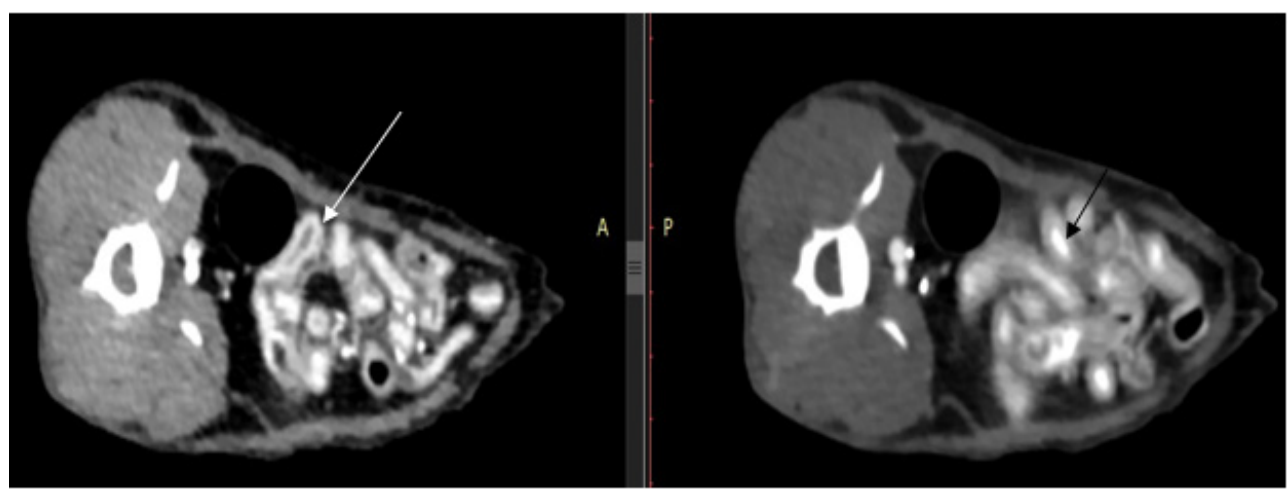

Fig. 3. Post contrast arterial time and tardive venouse coronal view of the small intestine, presence of the contrast agent in the intestinal wall (white arrow) and intestinal lumen (black arrow), 110kV, $22 \mathrm{~mA}$

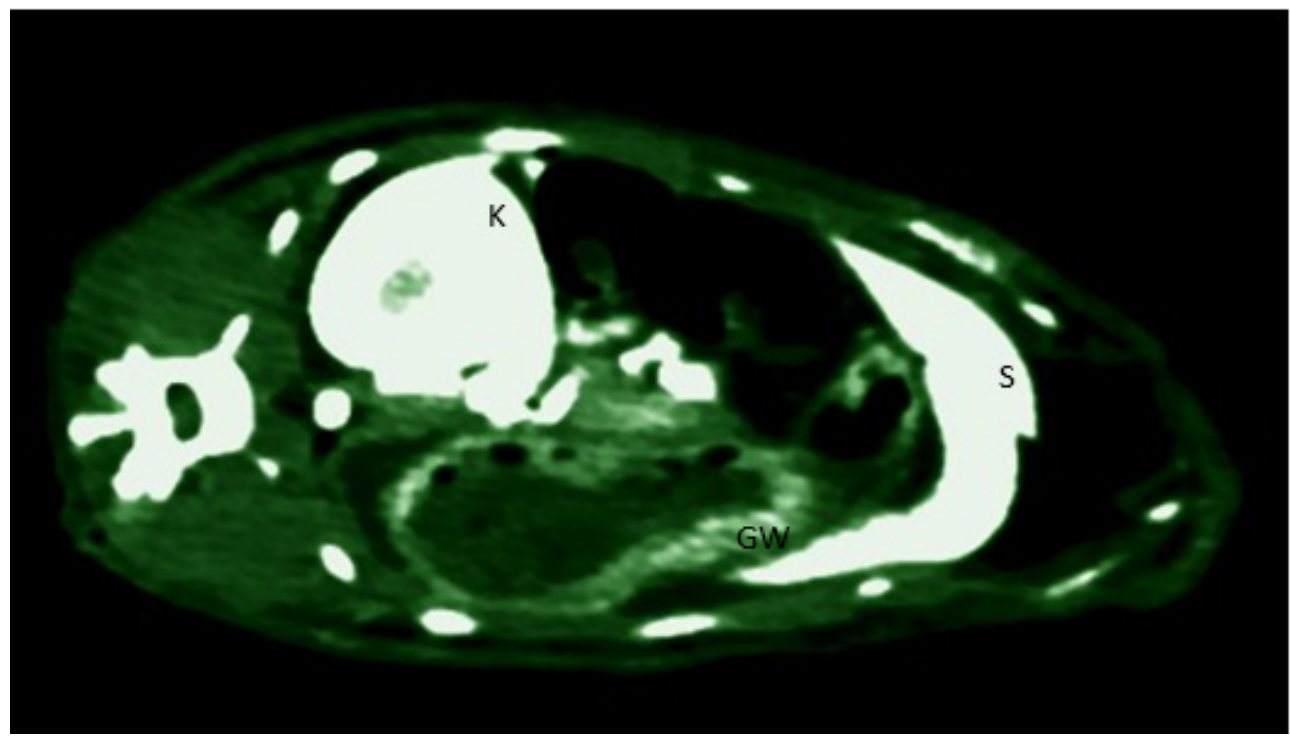

Fig. 4. Arterial time, highlight of kidney (K), spleen (S) and gastric wall (GW). 


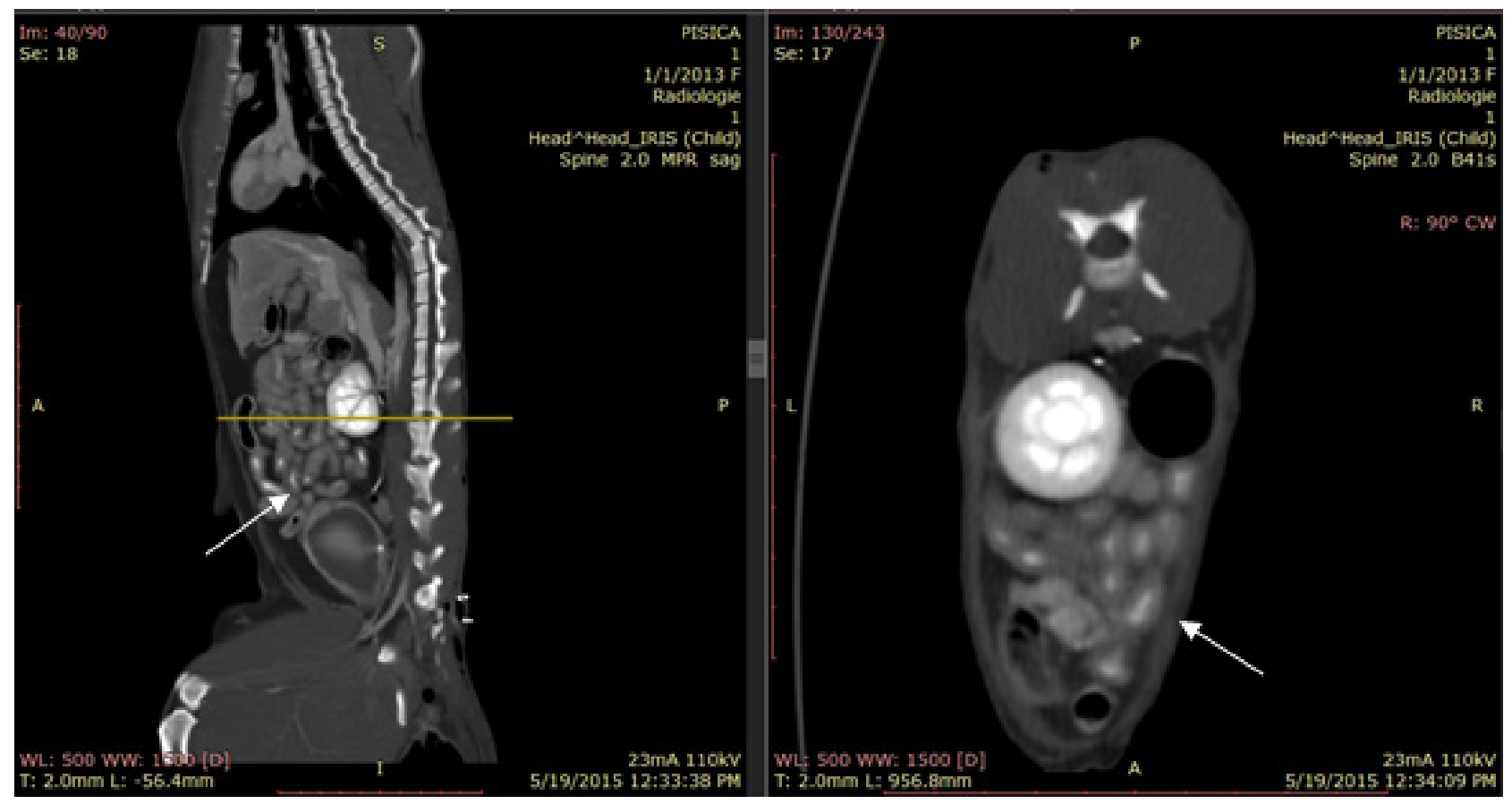

Fig. 5. Late venouse time, sagittal and coronal view of the GI tract. Contrast agent persist in the intestinal lumenum

and in the tardive contrast phase the contrast agent is evident in the intestinal lumen (fig. 2, fig. 3).

In the arterial phase the contrast agent is present in the spleen, kidney but also in the gastric wall (fig. 4), that suggest a hyperaemia of the stomach wall.

We have excluded a possible internal hemorragia or free blood in the abdomen, because the contrast agent was contained in the organs and GI tract.

In the venous late phase, after approximatively 15 minute post contrast administration, the contrast agent is evident in the venous system, in the kidney pelvis but a small quantity of contrast substance is still evident in the intestinal lumen (fig. 5).

Presence of the contrast agent in the intestinal lumen suggest passing of the blood through the intestinal wall because on intestinal hemorrhagia. In normal situation the blood with contrast agent should be visible only inside the blood vessels not inside the intestinal lumen (Macari and Balthazar, 2001).

\section{CONCLUSION}

Contrast CT examination proved to be extremely useful and revealed an accumulation of the contrast agent in the intestinal lumen due to intestinal bleeding which explain the palness of the mucosa in our case. Radiography and ultrasonography have not proven reliable in this case for a diagnostic of GI internal bleeding.

\section{REFERENCES}

1. Bae KT (2010) Intravenous contrast medium administration and scan timing at CT: considerations and approaches. Radiology 256:32-61.

2. Burk RL (1992). Computed tomographic imaging of nasal disease in 100 dogs. Vet Radiol Ultrasound 33: 177-180.

3. Boysen SR, Tidwell AS, Penninck DG (2003). Ultrasonographic findings in dogs and cats with gastrointestinal perforation. Veterinary Radiology \& Ultrasound 44(5):556-564.

4. Grassi R, Pinto A, Rossi G, Rotondo A (1998). Conventional plain-film radiology, ultrasonography and CT in jejunoileal perforation. Acta Radiologica 39:52-56.

5. Heng HG, Wrigley RH, Kraft SL, Powers BE (2005). Fat is responsible for an intramural radiolucent band in the feline stomach wall. Veterinary Radiology \& Ultrasound 46 (1):54-56.

6. Kleine JL, Lamb C (1989). Comparative organ imaging: the gastrointestinal tract. Veterinary Radiology 30(3):133141. 
7. Macari M, Balthazar EJ (2001). CT of bowel wall thickening. Significance and pitfalls of interpretation. American Journal of Roentgenology 176(5):1105-1123.

8. Makara M, Chau J, Hall E, Kloepperl H, Podadera J, Barrs V (2015). Effects of two contrast injection protocols on feline aortic and hepatic enhancement using dynamic computed tomography. Veterinary Radiology \& Ultrasound 56(4):367-373.

9. Moore MP, Gavin PR, Kraft SL, Dehaan CE, Leathers CW, Dorn RV (1991). M. R. I., C. T. and clinical features from four dogs with nasal tumor. Vet Radiol Ultrasound 32:1925.
10. Patsikas MN, Rallis T, Kladakis SE, Dessiris AK (2001). Computed tomography diagnosis of isolated splenic torsion in a dog. Vet Radiol Ultrasound 42:235-237.

11. Plummer SB, Wheeler SJ, Thrall DE, Kornegay JN (1992). Computed tomography of primary inflammatory brain disorders in dogs and cats. Vet Radiol Ultrasound 33:307315.

12. Schwarz T, Saunders J et al (2011). Veterinary computed tomography. Wiley-Blackwell, Oxford. 